\title{
Viable circulating tumour cell detection using multiplex RNA in situ hybridisation predicts progression-free survival in metastatic breast cancer patients
}

\author{
RE Payne', F Wang', N Su${ }^{2}$, J Krell ${ }^{3}$, A Zebrowski ${ }^{3}$ E Yagüe', X-J Ma ${ }^{2}$, Y Luo ${ }^{2}$ and RC Coombes*, \\ 'Division of Cancer, Imperial College, MRC Cyclotron Building, Hammersmith Hospitals Trust, Hammersmith Hospital, Du Cane Road, London WI2 \\ ONN, UK; ${ }^{2}$ Advanced Cell Diagnostics, Inc., 26229 Eden Landing Road, Hayward, CA 94545, USA; ${ }^{3}$ Department of Medical Oncology, Charing Cross \\ Hospital, London W6 8RF, UK
}

BACKGROUND: Current approaches for detecting circulating tumour cells (CTCS) in blood are dependent on CTC enrichment and are based either on surface epithelial markers on CTCs or on cell size differences. The objectives of this study were to develop and characterise an ultrasensitive multiplex fluorescent RNA in situ hybridisation (ISH)-based CTC detection system called CTCscope. This method detects a multitude of tumour-specific markers at single-cell level in blood.

METHODS: Healthy blood samples spiked with tumour cell lines were used as a model system for the development and initial characterisation of CTCscope. To demonstrate the feasibility of CTC detection in patient blood, duplicate blood samples were drawn from 45 metastatic breast cancer patients for analysis by CTCscope and the CellSearch system. The association of CTCs with the tumour marker CAI5-3 and progression-free survival (PFS) were assessed.

RESULTS: CTCscope detected CTC transcripts of eight epithelial markers and three epithelial-mesenchymal-transition (EMT) markers for increased sensitivity. CTCscope was used to detect CTCs with minimal enrichment, and did not detect apoptotic or dead cells. In patient blood samples, CTCs detected by CellSearch, but not CTCscope, were positively correlated with CAI5-3 levels. Circulating tumour cells detected by either CTCscope or CellSearch predicted PFS (CTCscope, HR (hazard ratio) 2.26, 95\% Cl 1.18-4.35, $P=0.014$; CellSearch, HR 2.50, 95\% Cl I.27-4.90, $P=0.008$ ).

CONCLUSION: CTCscope offers unique advantages over existing CTC detection approaches. By enumerating and characterising only viable CTCs, CTCscope provides additional prognostic and predictive information in therapy monitoring.

British Journal of Cancer (2012) 106, 1790-1797. doi: I0.1038/bjc.20 I2.137 www.bjcancer.com

Published online 26 April 2012

(c) 2012 Cancer Research UK

Keywords: circulating tumour cells; breast cancer; mRNA; in situ hybridisation

Circulating tumour cells (CTCs) are shed into the bloodstream from primary and metastatic solid tumours and are seen as important emerging biomarkers of cancer (Smith et al, 2000; Cristofanilli, 2006). Sensitive and specific detection of CTCs is crucial to enable their predictive and prognostic use. There is evidence to suggest current methods do not detect all CTCs present in a blood sample and may miss CTCs with low expression of commonly used epithelial markers such as EpCAM or the cytokeratin family (Nagrath et al, 2007; Punnoose et al, 2010). Further evidence suggests that CTCs associated with ER-negative breast cancers that express a putative tumour stem cell phenotype (often associated with aggressive disease), are not detected (Sieuwerts et al, 2009). As it is hoped that CTCs will provide information for personalised therapy, it is important to be able to detect the full heterogeneity present in a CTC population by detecting a number of tumour markers. This is particularly important in early stage cancer where CTCs are very rare and their characterisation could enable the potential eradication of

*Correspondence: Professor RC Coombes;

E-mail: c.coombes@imperial.ac.uk

Received 3 January 2012; revised I March 2012; accepted 21 March 2012; published online 26 April 2012 micrometastases by the use of specific therapies. Single-cell detection and characterisation is limited owing to the technical difficulties of isolating adequate numbers of cells from the blood as well as the problems (both cost and optimisation time) in multiplexing antibodies against multiple tumour markers. Antibody staining is also prone to low specificity and user subjectivity when interpreting the staining (Gown, 2008).

The use of quantitative reverse transcription real-time PCR (qRT-PCR) for detecting multiple tumour marker mRNAs from blood is a highly sensitive method. One drawback is the detection of illegitimate transcripts present in blood cells, and it also does not account for tumour cell heterogeneity. The use of a method to detect tumour marker mRNAs in single CTCs in situ is therefore attractive. However, the use of RNA in situ hybridisation (ISH) in CTCs has drawn little attention owing to limited sensitivity and specificity of conventional RNA ISH methods. Recently, an ultrasensitive and specific multiplex RNA ISH technology, RNAscope, was developed, which is capable of single RNA molecule detection (Ukpo et al, 2011; Wang et al, 2012). This technology can detect multiple transcripts simultaneously in two ways: the target probes for different genes can have the same recognition sequence for the signal amplification system, thus generating a 'pooled' signal; alternatively, multiple independent signal amplification 
systems can be used to simultaneously detect multiple target RNA species each as a distinct signal. This unique feature of the technology makes it well suited for CTC identification and characterisation. Here, we describe the development and characterisation of CTCscope using a panel of epithelial and tumour mRNA markers and demonstrate its use in the detection of single viable CTCs from metastatic breast cancer patients and its correlation with progression-free survival.

\section{MATERIALS AND METHODS}

\section{Study design}

Breast cancer patients with metastatic disease were recruited prospectively and sequentially from Charing Cross Hospital, London. The study had been approved by the Local Ethics Committee. All patients provided written, informed consent. The primary tumour characteristics of these patients are detailed in Table 1 . Patients were included if they had histologically confirmed

Table I Clinical characteristics of metastatic breast cancer patients

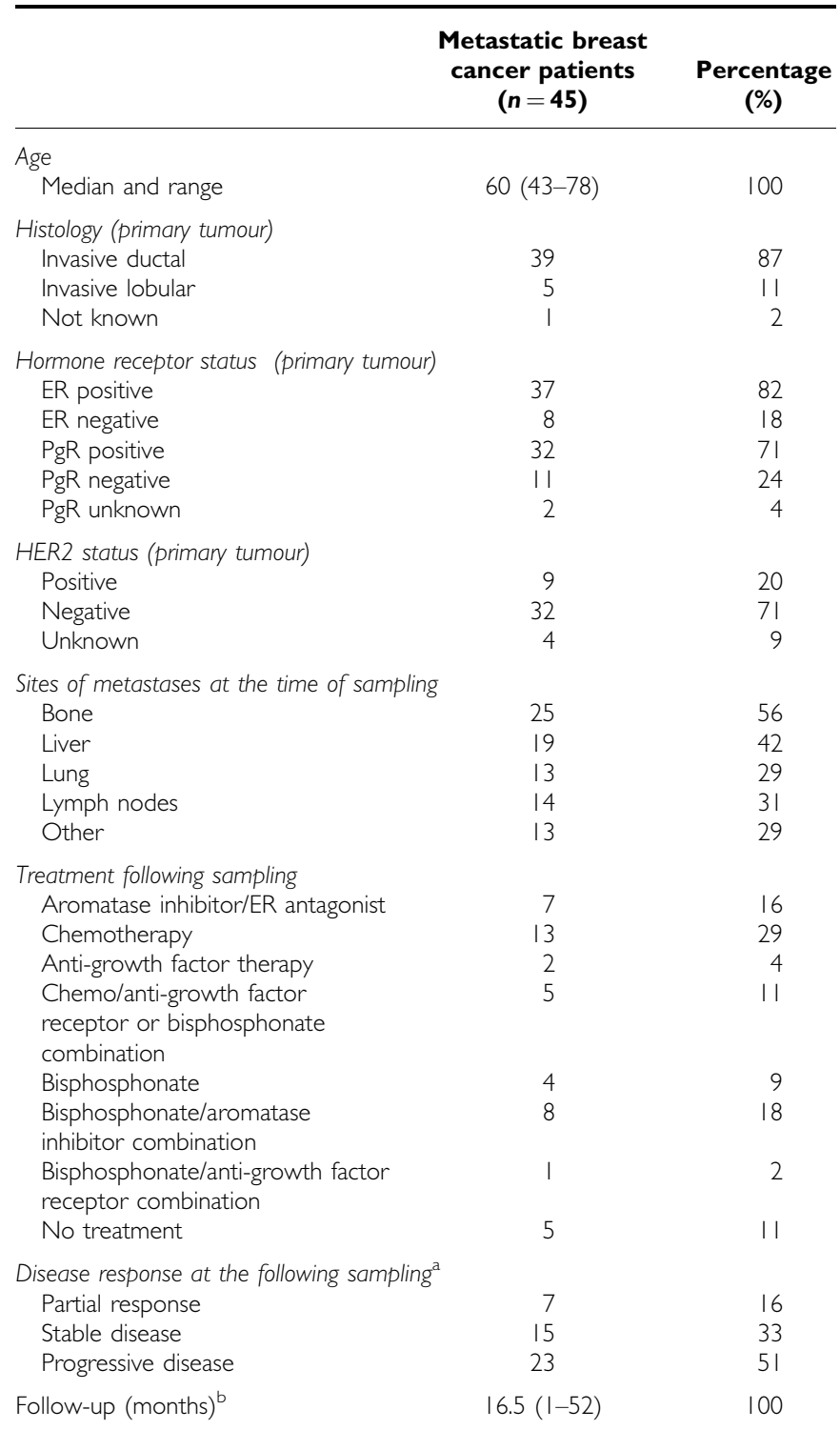

Abbreviations: $E R=$ oestrogen receptor; $\mathrm{PgR}=$ progestrone receptor. ${ }^{\mathrm{a}}$ Response

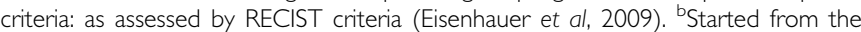
time of blood sampling for circulating tumour cell analysis. evidence of metastatic breast cancer. Clinical examination and either Computed Tomography or Magnetic Resonance Imaging scans were performed at the start of the study (time of blood sampling) and then every 6 weeks following sampling. Disease progression status was assessed using the RECIST (Response Evaluation Criteria for Solid Tumours) (Therasse et al, 2000; Eisenhauer et al, 2009).

Control blood samples from healthy donors were collected at the Stanford Blood Center (Palo Alto, CA, USA).

\section{Statistical analysis}

Progression-free survival (PFS) was the interval from the date of blood sampling to the date of clinical progression or death due to any cause. PFS differences according to CTC status (presence or absence) and CA15-3 levels (using median as the cutoff) were computed using the Kaplan-Meier method and compared by logrank test. Univariate Cox proportional hazard regression analysis was used to assess prognostic performance of CTC detection and CA15-3 levels. Concordance of CTC detection (present or absent) between CTCscope and CellSearch was assessed by Fisher's exact test. The correlation between CA15-3 levels and CTC numbers was examined by Spearman rank correlation. All calculations were performed in the $\mathrm{R}$ statistical environment. All significance tests were two-sided, and a $P<0.05$ was considered statistically significant.

\section{The Cellsearch system}

Blood samples were collected in CellSave preservative tubes (Veridex LLC, Raritan, NJ, USA) and processed within $72 \mathrm{~h}$ of collection. The CellSearch system (Riethdorf et al, 2007) uses magnetic iron particles conjugated to antibodies against the epithelial cell adhesion molecule (EpCAM) to immunomagnetically enrich epithelial cells. The cells were labelled with the fluorescent nucleic acid dye 4',6-diamidino-2-phenylindole (DAPI); a mixture of two phycoerythrin-conjugated antibodies that bind to cytokeratins 8, 18 and 19 and a allophycocyanin-conjugated CD45 antibody (to distinguish CTCs from leukocytes). A control consisting of SK-BR-3 cells was also used in each run to ensure the efficiency and specificity of detecting a low and high population of CTCs (Veridex LLC). Briefly, each $7.5 \mathrm{ml}$ sample of blood was mixed with $6.5 \mathrm{ml}$ of buffer and centrifuged at $800 \mathrm{~g}$ for $10 \mathrm{~min}$, and then placed in the Autoprep system. The cells were then aspirated into the MagNest cell presentation device (Veridex LLC). This device is made up of a chamber and two magnets that hold the immunomagnetically labelled cells to the surface of a cartridge for analysis using the CellTracks Analyser.

\section{Measurement of serum CA15-3}

CA15-3 was measured in serum using the Abbott Architect Ca 15-3 kit. This uses both 115D8 and DF3 monoclonal antibodies; the assay has a sensitivity of $<0.5 \mathrm{U} \mathrm{ml}^{-1}$ and has been extensively described in the literature (Hayes et al, 1986).

\section{Method for spiking cancer cells in blood}

To spike rare numbers $(<100)$ of tumour cells in blood, a suitable concentration of cultured cancer cells (e.g. MDA-MB-468) were

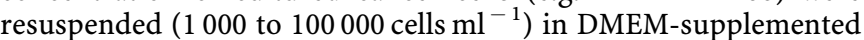
media. One $\mu \mathrm{l}$ of cell suspension was transferred to the corner of a glass coverslip. The cells were then enumerated using a tally counter under $\times 10$ magnification on a bright field microscope with the entire $1 \mu \mathrm{l}$ drop in the field of view. The entire drop of cell suspension was then transferred into $5 \mathrm{ml}$ of blood of healthy donor in a blood collection tube by flushing four times with $50 \mu \mathrm{l}$ $1 \times$ phosphate-buffered saline (PBS). All the residual PBS on the 
slide was also transferred to the tube to minimise cell loss. The blood tube was then inverted 5-10 times with the lid on.

\section{Peripheral blood mononuclear cell (PBMC) preparation for CTCscope}

Peripheral blood $(7.5 \mathrm{ml})$ was drawn into a vacutainer with EDTA and processed within $6 \mathrm{~h}$. Peripheral blood mononuclear cells were then prepared via Ficoll-gradient using Accuspin system-histopaque1077 tubes (Sigma-Aldrich, St Louis, MO, USA) according to the manufacturer's manual, and fixed in $10 \%$ buffered formaldehyde solution at $37^{\circ} \mathrm{C}$ for $40 \mathrm{~min}$ with rotation. The cells were then washed and stored in $700 \mu \mathrm{l} 70 \%$ ethanol at room temperature. Metastatic patient blood samples were processed according to the same protocol and stored for up to a month before CTCscope analysis.

\section{CTCscope assay}

In situ hybridisation probes were designed to target CK5, CK6, CK8, CK14, CK17, CK18, CK19, CK20, EpCAM, MUC1, VIM, TWIST, CDH2 (N-Cadherin), FN1 (fibronectin) and CD45 mRNAs, respectively, using a computer algorithm described earlier (Bushnell et al, 1999) (see Supplemental Table 2 for target regions). Each oligo probe has an average length of 25 nucleotides in the target recognition region and is designed to have a Tm within a defined range so that all the target probes will hybridise optimally under the assay conditions. For CTCscope staining, formaldehyde-fixed cells were spun onto SuperFrost Plus glass slides using a Hettich cyto-centrifuge and air dried. Typically, twothirds of the preparation ( $\sim 5 \mathrm{ml}$ blood) was cytospun onto slides for CTCscope analysis. The samples were then processed with the RNAscope assay described previously (Wang et al, 2012). Briefly, the cells were treated with protease followed by incubation with the target probes at $40^{\circ} \mathrm{C}$ for $3 \mathrm{~h}$. The signal is amplified by a series of nucleic acid hybridisation events that result in accumulation of a large number of oligonucleotide probes conjugated with Alexa fluorescent dyes (Wang et al, 2012). Different target probes can be pooled and labelled with the same fluorescent colour, or with different colours in a multiplex assay. Nuclei were stained with 4',6-diamidino-2-phenylindole, dihydrochloride (DAPI).

\section{Circulating tumour cell enumeration and imaging}

Circulating tumour cells were enumerated manually under $\times 20$ magnification on a Zeiss (New York, NY, USA) Axioplan M1 microscope, using the Alexa488 channel (Life Technologies, Carlsbad, CA, USA) (panCTC staining). A sample was qualified for CTC scoring if the majority of PBMCs stained positively with CD45 (Alexa546 channel, Life Technologies), indicating acceptable RNA integrity. A CTC was identified by strong positive panCTC mRNA staining, positive nuclear staining (DAPI channel), and no CD45 mRNA signal. Circulating tumour cell images were acquired with the Nuance Multispectral Imaging System (CRI, Cambridge, MA, USA) using a combination of long-pass filter sets: DAPI $(425 \mathrm{~nm} / 475 \mathrm{~nm})$, Alexa488 $(470 \mathrm{~nm} / 515 \mathrm{~nm})$, and Alexa546 $(535 \mathrm{~nm} / 590 \mathrm{~nm})$. Overlapping signals from different fluorophores were separated by comparing composite signals to a reference spectral library generated with single colour stained samples.

\section{CTCscope/antibody double staining}

MDA-MB-468 cells were trypsinised, fixed, and stained according to the CTCscope protocol using pan-CK probes (CK8, CK18, and CK19) labelled with Alexa546. The samples were then blocked in $1 \times$ PBS with $1 \%$ BSA for $30 \mathrm{~min}$ at room temperature, and then incubated with M30 antibody (Roche, Indianapolis, IN, USA, cat. no. 12140322001) or Mouse anti-Cytokeratin antibody (Clone AE1/ AE3, Invitrogen Technologies, Carlsbad, CA, USA, cat. no.
08-4132) for $1 \mathrm{~h}$ at room temperature. The samples were washed and then incubated with FITC-conjugated goat anti-mouse antibody (Millipore, Billerica, MA, USA, cat no. 12-506) for $30 \mathrm{~min}$ at room temperature. Cell nuclei were stained with DAPI. Images were acquired with the Nuance Multispectral Imaging System (CRI) using the same filter set used for CTC analysis.

\section{RESULTS}

\section{The CTCscope assay}

The workflow of the CTCscope assay is shown in Figure 1A. CTCscope uses PBMC fractions obtained from whole blood using a Ficoll-gradient collection tube. This step provides limited enrichment by removing red blood cells and platelets but does not separate CTCs from white blood cells. Unlike other CTC detection methods, further enrichment from white blood cells is not required for this method owing to the highly sensitive and specific detection system employed. To evaluate the capability of CTCscope in the detection and characterisation of CTCs, probe sets against mRNAs encoding cytokeratin 8,18 , and 19 were combined (pan-CK) to detect epithelial cells. Another probe set was used on a separate colour to detect EGFR mRNA expression in CTCs. To determine whether rare cancer cells could be detected by CTCscope, cultured breast cancer cell lines (MCF7, SK-BR-3 and MDA-MB-468) were spiked into whole blood obtained from healthy individuals at approximately 50 cells per $10 \mathrm{ml}$ of blood. Peripheral blood mononuclear cells were collected and stained according to the CTCscope protocol. Spiked-in cells of all three cell lines could be identified by strong pan-CK staining, whereas the surrounding PBMCs showed minimal fluorescent signals (Figure 1B). In addition, MCF7, SK-BR-3, and MDA-MB-468 cells showed different EGFR mRNA expression levels, with MDA-MB-468 having the highest level of EGFR transcripts, SK-BR-3 at a medium level, and the majority of MCF7 cells having no EGFR mRNA expression (Figure 1B). These results are consistent with the known EGFR protein expression status in these cell lines (Kaplan et al, 1990; Modjtahedi et al, 1993; Wang et al, 1999) demonstrating CTCscope's molecular profiling capability.

Given that cancer cells with different origins or at different progression stages have varied expression levels of cytokeratins and other epithelial cell markers, we incorporated additional target probes into our CTC detection system to further enhance its sensitivity. The expanded CTC panel (panCTC) included traditional epithelial cell markers (cytokeratins 8, 14, 17, 18, 19, and 20, EpCAM, and MUC-1) and three genes expressed in tumour cells that have undergone EMT) (Yang et al, 2004; Yang et al, 2007). All these markers were independently tested by staining PBMCs purified from blood with spiked-in cells of MDA-MB-468 and MRC-5 (a lung fibroblast cell line expressing Twist, N-Cadherin, and fibronectin), and they were found to be specific to the spikedin cells and were not detectable in background PBMCs (Supplementary Figure 1A and B). Several other epithelial and EMT markers, including cytokeratin 5, 6, and vimentin, were also tested, but they were excluded from the CTC detection panel owing to their expression in blood cells (Supplementary Figure 1C). A negative selection marker (CD45) was also developed to ensure that no leukocytes were counted as CTCs. CD45 also served as an RNA quality indicator, and only those samples with CD45 RNA staining in PBMCs were qualified for subsequent CTC screening. A CTC was identified as a nucleated (DAPI-positive) cell with positive staining of CTC markers but no staining for CD45.

To determine whether the expanded panel of CTC markers (panCTC) could lead to higher detection sensitivity compared with pan-CK markers (CK8, CK18, and CK19), MDA-MB-468 cells were hybridised with probes to detect CK19, pan-CK, and panCTC markers, respectively. As expected, an increase in fluorescent signal was observed when the number of combined probes 
A

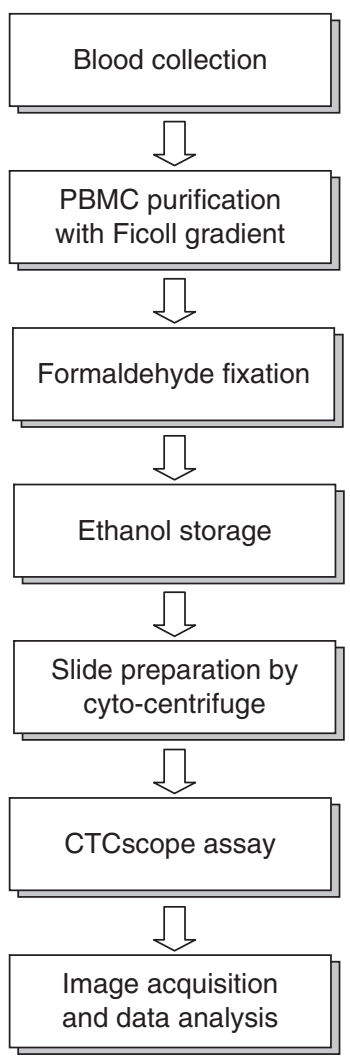

B
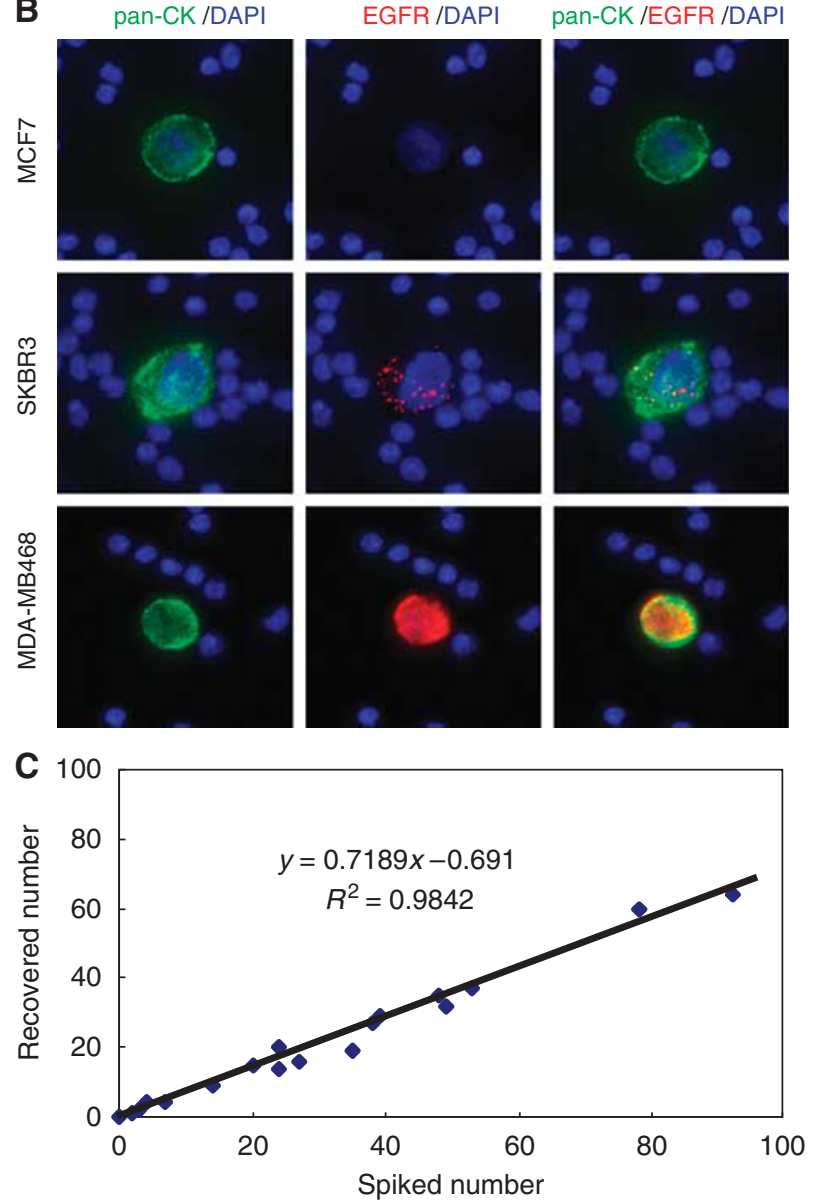

Figure I The CTCscope assay. (A) CTCscope workflow from blood sample collection to image acquisition and data analysis. (B) Ficoll-prepared human PBMCs spiked with MCF7, SK-BR-3, or MDA-MB-468 tumour cells were hybridised with a pooled probe set for pan-CK (CK8, CKI 8, and CK19) and EGFR mRNAs. Merged images are shown in the right column. Cells were counterstained with DAPI (blue). (C) Efficient cell recovery by the CTCscope. Low numbers of MDA-MB-468 cells were spiked into $5 \mathrm{ml}$ blood, PBMCs were enriched and processed by CTCscope, and the number of cells recovered by CTCscope plotted against the number of spiked cells.

increased (Supplementary Figure 1D). We next confirmed that the increased sensitivity of the panCTC panel did not result in a decrease in specificity: blood samples from 13 healthy volunteers were analysed by CTCscope, and no panCTC-positive cells were identified.

To assess the recovery rate and assay linearity of CTCscope, a breast cancer cell line (MDA-MB-468) was spiked at different concentrations (1-92 cells per $5 \mathrm{ml}$ ) into blood from healthy donors. The PBMCs were collected and CTCscope performed. The average recovery rate was $71 \% \pm 12 \%(n=17)$ (Figure $1 \mathrm{C}$ ).

Given that RNA degrades quickly upon apoptosis, we hypothesised that CTCscope should only detect live CTCs but not dead or apoptotic cells (at the time of fixation). To test this, we performed a CTCscope assay on MDA-MB-468 cells, followed by immunostaining with M30, an epithelial cell apoptosis marker antibody (Leers et al, 1999) or pan-CK antibody. A population of naturally occurring apoptotic cells could be identified by their positive M30 staining; consistent with our hypothesis. These M30-positive apoptotic cells had undetectable pan-CK RNA expression (Figure 2A, white arrows). Note that all cells stained positively for either pan-CK RNA or M30 but not both, suggesting that an apoptotic CTC cannot be stained by RNAscope. In contrast, the pan-CK antibody stained all cells including those with low or no pan-CK RNA staining (Figure 2B), suggesting that pan-CK protein staining could not distinguish live cells from cells undergoing apoptosis.

\section{CTCscope analysis of blood samples from breast cancer patients}

We next wanted to demonstrate whether the CTCscope assay could be used to detect CTCs in patients' blood. In all, 45 unselected breast cancer patients with metastatic breast cancer were recruited over a 5-month period from a single institution. Of the 45 patients, $40(89 \%)$ received cytotoxic, hormonal, biological or bisphosphonate treatments and $5(11 \%)$ received no treatment following blood sampling. In all, 23 patients $(51 \%)$ had progressive disease, $15(33 \%)$ had stable disease, and $7(16 \%)$ had a partial response according to RECIST criteria (Therasse et al, 2000; Eisenhauer et al, 2009) during the follow-up period of a median of 16 weeks. All patients had duplicated blood samples taken for direct comparison between the CellSearch system and CTCscope (see Supplemental Table 1 for the number of CTCs detected for each patient). Approximately $5 \mathrm{ml}$ of blood was analysed by CTCscope, whereas $7.5 \mathrm{ml}$ was processed by CellSearch.

After staining by CTCscope, candidate CTCs were identified by screening at low magnification (Figure 2C) for positive panCTC marker staining. High magnification images were then taken to confirm the identification of CTCs by the absence of CD45 staining. In general, the CTCs identified in the patients could be categorised according to cell size. In some patients, CTCs had a similar or slightly larger size than PMBCs (Figure 2D), whereas in other patients, the CTCs were significantly larger (Figure 2E). It is 

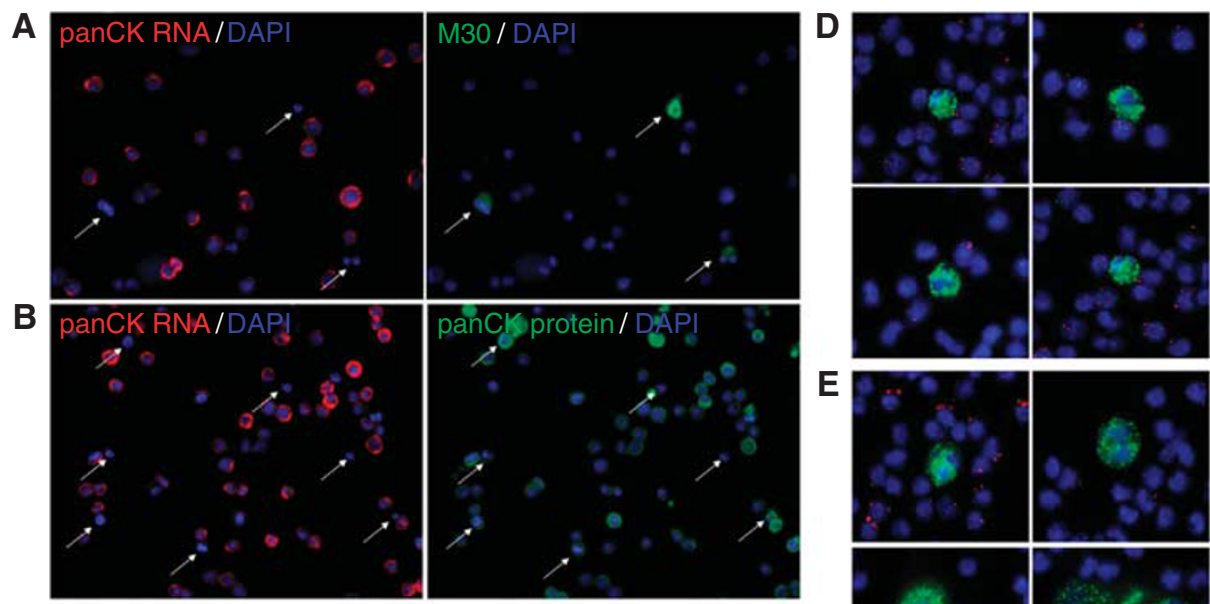

E
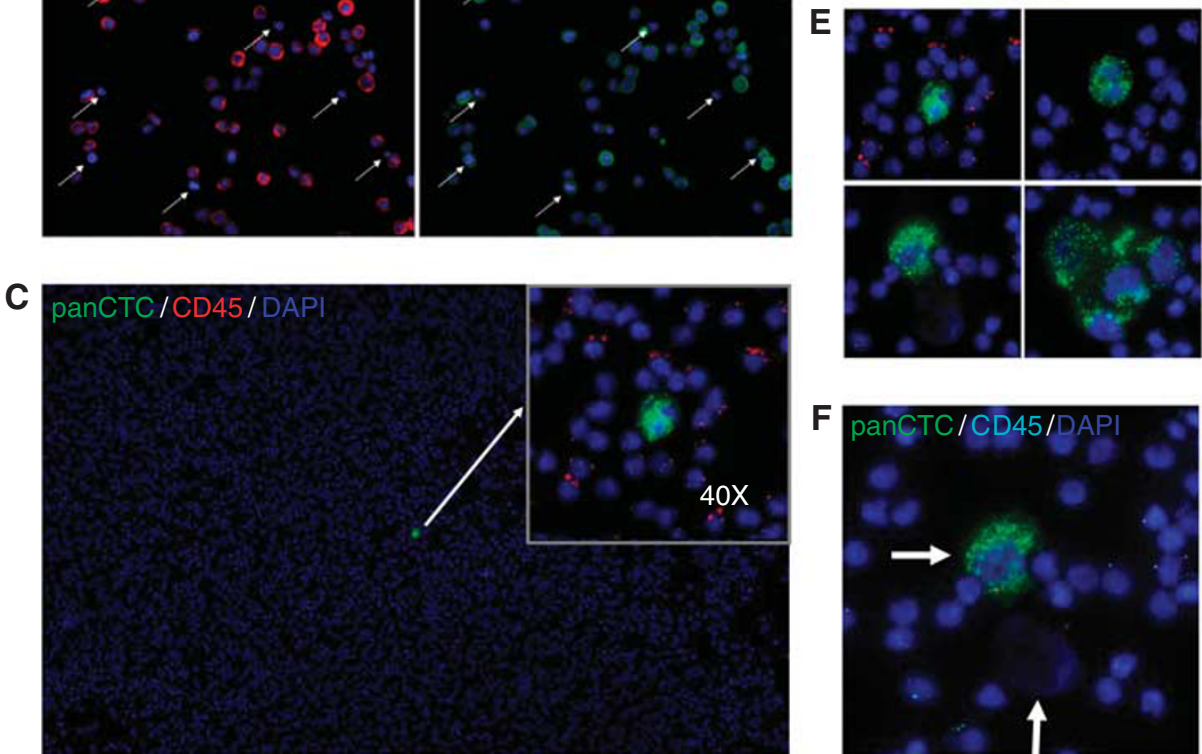

Figure 2 Specific identification of non-apoptotic cells by CTCscope and examples of CTCs in metastatic breast cancer patients. (A) MDA-MB-468 cells were hybridised with a pooled probe set for pan-CK (CK8, CK I 8, and CK 19), red signal, followed by immunostaining with M30 antibody (green). (B) MDAMB-468 cells were hybridised to pan-CK probes (red) followed by immunostaining with pan-CK antibody (green). Cells were counterstained with DAPI (blue). Arrows indicate apoptotic cells showing signal for CK protein but not for mRNA. (C) Peripheral blood mononuclear cell preparations from a metastatic breast cancer patient were hybridised with a pooled probe set for pan-CK (CK8, CKI8, and CKI9; green) and CD45 (red) mRNAs and counterstained with DAPI. A CTC was initially identified at $\times 10$ magnification and then confirmed by its absence of CD45 mRNA signals at $\times 40$ magnification (insert). (D) Example images of patient CTCs that have similar size as PBMCs. (E) Example images of patient CTCs that were significantly larger than PBMCs. Both $(\mathbf{D})$ and $(\mathbf{E})$ at $\times 40$. (F) Two CTCs (arrows) from one metastatic breast cancer patient. One of the CTCs stained strongly with panCTC mRNAs, whereas the other completely lacked any mRNA signal.

worth noting that we also observed some cells with apparent tumour cell morphology (e.g. large nuclei) or tumour cell clusters which showed no panCTC marker staining (Figure $2 \mathrm{~F}$ ). It is possible that these CTCs had undergone apoptosis and were therefore not detectable by CTCscope. CTCscope and CellSearch detected at least one CTC in similar proportions of patients: CTCscope detected CTCs in 21 out of 45 patients $(47 \%)$ and the CellSearch system detected CTCs in 23 out of 45 patients (51\%). The two methods had concordant results for 31 of the 45 patients (69\%; $P=0.017$, Fisher's exact test); 15 patients (33\%) were positive for CTCs by both methods and 16 patients (36\%) were negative for CTCs by both methods. A proportion of patients had CTCs detected by only one method, $8(18 \%)$ by the CellSearch system and $6(13 \%)$ by CTCscope. A total of 29 out of $45(64 \%)$ patients showed the presence of CTCs by one or both methods. The CellSearch system detected far more cells than CTCscope with a mean of 19.53 compared with 1.56 and a median of 1 compared with 0 CTCs per $7.5 \mathrm{ml}$ of blood (Table 2).

\section{Detection of CTCs by CTCscope predicts PFS}

The CTC status for each patient was defined as either positive (at least one CTC detected) or negative (no CTC detected). Progression-free survival differed significantly according to CTC status by either CTCscope (log-rank $P=0.014$ ) or CellSearch $(P=0.006)$ with positive CTC status associated with shorter PFS
(Figure 3). CellSearch results were also significantly associated with PFS when the established cutoff of 5 CTCs for CellSearch (Cristofanilli, 2006) was used ( $P=0.013$; Figure 3$)$. Consistent with being a prognostic factor in breast cancer, CA15-3 levels also significantly predicted PFS $(P=0.004$; Figure 3$)$.

We also performed univariate Cox regression to further evaluate the association of CTC status with PFS (Table 3). Circulating tumour cell status by CellSearch had a slightly increased association with PFS (HR 2.50, 95\% CI 1.27-4.90, $P=0.008$ ) than that by CTCscope (HR 2.26, 95\% CI 1.18-4.35, $P=0.014$ ). In addition, serum CA 15-3 levels had a stronger correlation with CTC numbers by CellSearch (Spearman rho $=0.52, P=0.0003$ ) than by CTCscope (rho $=0.24, P=0.09)$ (Table 3).

\section{DISCUSSION}

Circulating tumour cells (CTCs) are extremely rare in the blood at around CTC per billion blood cells, even in metastatic cancer patients. As a result, their detection and characterisation remains a significant technical challenge. Here, we have described a novel RNA ISH assay, CTCscope, for both the detection and molecular analysis of CTCs. By using cell line models, we demonstrated that CTCscope is highly sensitive and specific for CTC detection in blood samples without special enrichment. This is a significant feature of CTCscope because current enrichment methods, such as 
Table 2 Comparison of CTC detection by the CellSearch system and CTCscope in paired blood samples from 45 patients with metastatic breast cancer

\begin{tabular}{|c|c|c|c|}
\hline & $\begin{array}{l}\text { CellSearch } \\
\text { system }\end{array}$ & & CTCscope \\
\hline Patients positive for $\operatorname{CTCs}^{\mathrm{a}}(n=45)$ & $23(5 \mid \%)$ & & $21(47 \%)$ \\
\hline $\begin{array}{l}\text { Patients with CTCs detected by both } \\
\text { methods }^{\mathrm{a}}\end{array}$ & & $15(33 \%)$ & \\
\hline $\begin{array}{l}\text { Patients with CTCs detected by either } \\
\text { method }^{\mathrm{a}}\end{array}$ & & $16(36 \%)$ & \\
\hline Concordance ( $P$-value) & & $0.017^{\mathrm{b}}$ & \\
\hline $\begin{array}{l}\text { Patients with CTCs detected with one } \\
\text { method only }\end{array}$ & $8(18 \%)$ & & $6(13 \%)$ \\
\hline Mean no. CTCs & 19.53 & & 1.56 \\
\hline $95 \% \mathrm{Cl}$ & $6.33-32.73$ & & $0.4-2.71$ \\
\hline Median & 1 & & 0 \\
\hline Range & $0-223$ & & $0-23$ \\
\hline
\end{tabular}

Abbreviations: $\mathrm{Cl}=$ confidence interval; $\mathrm{CTC}=$ circulating tumour cells. ${ }^{\text {PPositive for }}$ CTCs when one or more CTCs present in $7.5 \mathrm{ml}$ blood. 'Statistically significant (Fisher's exact test)
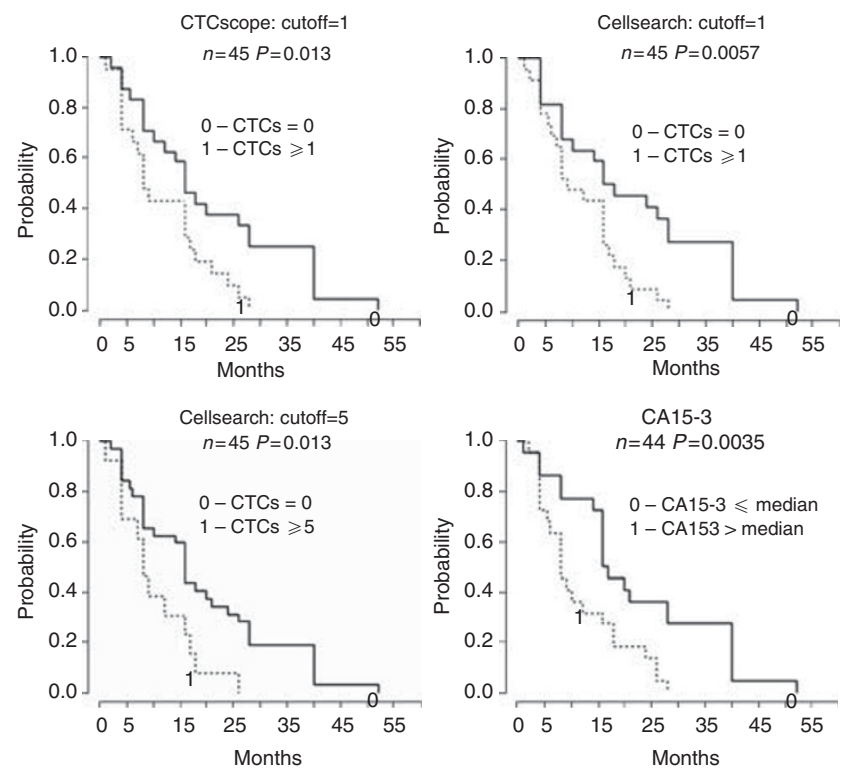

Figure 3 Kaplan-Meier plots of progression-free survival according to CTC status at time of sampling. Kaplan-Meier survival curves were drawn for patients with positive (dashed line) or negative (solid line) CTC status according to the cutoffs as indicated. For CAI5-3, the median was used as the cutoff.

EpCAM-based immuno-enrichment and cell size-based filtration, do not detect all CTCs due to the significant heterogeneity in antigen expression and cell size. The ability to detect CTCs expressing low levels of classical epithelial markers, such as CKs and EpCAM, was enhanced in CTCscope in two ways. Firstly, the panCTC gene panel included a cocktail of eight epithelial markers, increasing the sensitivity of detecting epithelial marker expression. Secondly, the panCTC panel included three markers of EMT. Evidence of co-expression of epithelial and mesenchymal markers in CTCs has been shown (Armstrong et al, 2011), however CTCs with EMT characteristics can have low or undetectable levels of CK/EpCAM expression and are thus not effectively detected by methods relying on epithelial markers alone (Lecharpentier et al, 2011; Raimondi et al, 2011). These cells have been attracting increasing attention in CTC research owing to their ability to invade and seed metastasis on one hand and to evade chemotherapy on the other (Bonnomet et al, 2010). In addition, using cell line models, we showed that unlike protein marker-based detection
Table 3

Correlation of CTC detection with PFS and CAI5-3

\begin{tabular}{cccccc}
\hline \multicolumn{3}{c}{ Univariate Cox regression } & & & \multicolumn{2}{c}{ Correlation with CA I5-3 } \\
\cline { 1 - 2 } \cline { 5 - 6 } & HR (95\% Cl) & P-value & & Rho & P-value \\
\hline CellSearch (CTC $>0)$ & $2.50(1.27-4.90)$ & 0.008 & & 0.52 & 0.0003 \\
CTCscope (CTC $>0)$ & $2.26(1.18-4.35)$ & 0.014 & & 0.24 & 0.09 \\
\hline
\end{tabular}

Abbreviations: $\mathrm{Cl}=$ confidence interval; $\mathrm{CTC}=$ circulating tumour cell; $\mathrm{HR}=$ hazard ratio; $\mathrm{PFS}=$ progression-free survival.

methods, CTCscope only detected viable CTCs with intact RNA, excluding dead cells from enumeration. This is desirable because only the viable CTCs are likely to have metastatic potential. This property of CTCscope may be particularly useful in monitoring therapeutic response, where the number of surviving CTCs may be more indicative of disease progression and of emerging resistance to therapy than that of the total CTC population.

We also demonstrated efficacy of CTCscope in metastatic breast cancer patients by conducting a head-to-head comparison with CellSearch. This study showed CTCscope detected CTCs in $47 \%$ of patients compared with $51 \%$ of patient with CTCs by the CellSearch system using $5 \mathrm{ml} v s 7.5 \mathrm{ml}$ of blood. The concordance was high with 31 out of $45(69 \%)$ patients with results that concurred. The CellSearch system however, detected many more CTCs than CTCscope in most patients, with a mean of 19.53 compared with 1.56 and a median of 1 compared with 0 , respectively. The spiking experiments showed a $71 \%$ recovery rate, however, so it should therefore theoretically detect similar numbers of CTCs as the CellSearch system. This discrepancy may be due to loss of CTCs during Ficoll-gradient fractionation or damage to CTCs in patient blood by the pre-processing method used in CTCscope, whereas breast cancer cell lines may be more robust and homogeneous. Alternatively, a substantial proportion of CTCs in breast cancer patients may be dead, dying or in a quiescent or dormant state, and are thus 'invisible' to CTCscope, which requires presence of intact RNA. Evidence for this includes heterogeneity of the proliferation marker Ki-67 in CTCs, and also the finding of a short half-life of CTCs from prostate cancer patients suggesting a dying phenotype in the blood (Stott et al, 2010). The presence of an apoptotic marker has been found in CTCs, which also suggests a dying phenotype and may explain the discrepancy between the number of CTCs detected by CTCscope and by the CellSearch system (Rossi et al, 2010). Therefore, although CellSearch detects more CTCs, CTCscope detects clinically important viable, non-apoptotic cells, with intact mRNA.

We investigated whether CTC detection was prognostic of patient outcome in metastatic breast cancer patients. As expected, CTCs detected by CellSearch-predicted shorter PFS. Despite the much lower number of CTCs detected by CTCscope, the presence of at least one CTC also correlated significantly with shorter PFS in metastatic breast cancer patients. This highlights the functional importance of the cells detected with this technology. In addition, CTCs detected by CellSearch positively correlated with the serum tumour marker CA 15-3 levels but CTCs detected by CTCscope did not. This difference in correlation to CA15-3 between CTCscope and CellSearch may be due to their different ability to detect apoptotic/dead cells. CA15-3, derived from the MUC1 protein, is likely to be shed from both viable and apoptotic/dead cells (perhaps even more from the latter). This corresponds well to the inability of CellSearch to discriminate apoptotic/dead cells from viable cells. In contrast, the number of CTCs detected by CTCscope did not include apoptotic/dead cells and were thus less correlated with CA15-3 levels.

Multiplex reverse-transcription real-time PCR (qRT-PCR) is another approach to detect and characterise CTCs at the mRNA level (Van der Auwera et al, 2010; Strati et al, 2011). In this 
approach, partially or highly enriched CTCs are obtained for RNA extraction and analysis. Although qRT-PCR has high analytical sensitivity and specificity and is also quantitative, it has important limitations in CTC analysis. Firstly, it does not allow direct enumeration of CTCs. Secondly, the measured expression levels of tumour markers represent the 'averages' of a heterogeneous population of cells analysed. This lack of cellular resolution masks the heterogeneous nature of CTCs and leads to 'dilution' by non-CTCs. Thirdly, basal levels of expression of epithelial markers in blood cells can lead to false positive results. CTCscope avoids these issues by enabling single-cell level analysis. Because of the single molecule sensitivity of the underlying RNAscope technology, tumour marker expression levels can be quantified by counting the puncate fluorescent dots, which can be automated by image analysis algorithms.

Several aspects of CTCscope may be further improved. Firstly, using a more effective and gentle approach in pre-processing of blood samples to prevent damage to CTCs may further increase recovery of greater numbers of intact CTCs. Secondly, the search for relevant subpopulations of CTCs using additional markers may be of great importance, for example by improving detection of cancer stem cells. Thirdly, automated image analysis for CTC enumeration and characterisation will be highly desirable for increased throughput. Fully automated detection and analysis should be feasible for CTCscope, because false positive identification is minimal owing to

\section{REFERENCES}

Armstrong AJ, Marengo MS, Oltean S, Kemeny G, Bitting RL, Turnbull JD, Herold CI, Marcom PK, George DJ, Garcia-Blanco MA (2011) Circulating tumor cells from patients with advanced prostate and breast cancer display both epithelial and mesenchymal markers. Mol Cancer Res 9: 997-1007

Bonnomet A, Brysse A, Tachsidis A, Waltham M, Thompson EW, Polette M, Gilles C (2010) Epithelial-to-mesenchymal transitions and circulating tumor cells. J Mammary Gland Biol Neoplasia 15: 261-273

Bushnell S, Budde J, Catino T, Cole J, Derti A, Kelso R, Collins ML, Molino G, Sheridan P, Monahan J, Urdea M (1999) ProbeDesigner: for the design of probesets for branched DNA (bDNA) signal amplification assays. Bioinformatics 15: 348-355

Cristofanilli M (2006) Circulating tumor cells, disease progression, and survival in metastatic breast cancer. Semin Oncol 33: S9-S14

Eisenhauer EA, Therasse P, Bogaerts J, Schwartz LH, Sargent D, Ford R, Dancey J, Arbuck S, Gwyther S, Mooney M, Rubinstein L, Shankar L, Dodd L, Kaplan R, Lacombe D, Verweij J (2009) New response evaluation criteria in solid tumours: revised RECIST guideline (version 1.1). Eur $J$ Cancer 45: 228-247

Gown AM (2008) Current issues in ER and HER2 testing by IHC in breast cancer. Mod Pathol 21(Suppl 2): S8-S15

Hayes DF, Zurawski Jr VR, Kufe DW (1986) Comparison of circulating CA15-3 and carcinoembryonic antigen levels in patients with breast cancer. J Clin Oncol 4: 1542-1550

Kaplan O, Jaroszewski JW, Faustino PJ, Zugmaier G, Ennis BW, Lippman M, Cohen JS (1990) Toxicity and effects of epidermal growth factor on glucose metabolism of MDA-468 human breast cancer cells. J Biol Chem 265: 13641-13649

Lecharpentier A, Vielh P, Perez-Moreno P, Planchard D, Soria JC, Farace F (2011) Detection of circulating tumour cells with a hybrid (epithelial/ mesenchymal) phenotype in patients with metastatic non-small cell lung cancer. Br J Cancer 105(9): 1338-1341

Leers MP, Kolgen W, Bjorklund V, Bergman T, Tribbick G, Persson B, Bjorklund P, Ramaekers FC, Bjorklund B, Nap M, Jornvall H, Schutte B (1999) Immunocytochemical detection and mapping of a cytokeratin 18 neo-epitope exposed during early apoptosis. J Pathol 187: 567-572

Modjtahedi H, Styles JM, Dean CJ (1993) The human EGF receptor as a target for cancer therapy: six new rat mAbs against the receptor on the breast carcinoma MDA-MB 468. Br J Cancer 67: 247-253

Nagrath S, Sequist LV, Maheswaran S, Bell DW, Irimia D, Ulkus L, Smith MR, Kwak EL, Digumarthy S, Muzikansky A, Ryan P, Balis UJ, Tompkins RG, Haber DA, Toner M (2007) Isolation of rare circulating tumour cells in cancer patients by microchip technology. Nature 450: $1235-1239$ the high specificity of RNAscope ISH; in contrast, IHC-based detection methods require manual morphological examination to avoid false positives. Finally, further streamlining and automation of the protocol will be needed for increased robustness for eventual clinical adoption. In conclusion, CTCscope holds great promise in detecting and characterising the heterogeneity of CTCs for both clinical research and diagnostics.

\section{ACKNOWLEDGEMENTS}

We would like to thank David Krag and Ed Manna for their contribution in CTCscope development. This study was funded in part by Cancer Research UK as well as the Imperial BRC and ECMC (RCC), and National Cancer Institute R43/R44CA122444 (YL).

\section{Conflict of interest}

FW, NS, X-JM and YL hold stocks of Advanced Cell Diagnostics. The other authors declare no conflict of interest.

Supplementary Information accompanies the paper on British Journal of Cancer website (http://www.nature.com/bjc)
Punnoose EA, Atwal SK, Spoerke JM, Savage H, Pandita A, Yeh RF, Pirzkall A, Fine BM, Amler LC, Chen DS, Lackner MR (2010) Molecular biomarker analyses using circulating tumor cells. PloS One 5: e12517

Raimondi C, Gradilone A, Naso G, Vincenzi B, Petracca A, Nicolazzo C, Palazzo A, Saltarelli R, Spremberg F, Cortesi E, Gazzaniga P (2011) Epithelial-mesenchymal transition and stemness features in circulating tumor cells from breast cancer patients. Breast Cancer Res Treat 130: $449-455$

Riethdorf S, Fritsche H, Muller V, Rau T, Schindlbeck C, Rack B, Janni W, Coith C, Beck K, Janicke F, Jackson S, Gornet T, Cristofanilli M, Pantel K (2007) Detection of circulating tumor cells in peripheral blood of patients with metastatic breast cancer: a validation study of the CellSearch system. Clin Cancer Res 13: 920-928

Rossi E, Basso U, Celadin R, Zilio F, Pucciarelli S, Aieta M, Barile C, Sava T, Bonciarelli G, Tumolo S, Ghiotto C, Magro C, Jirillo A, Indraccolo S, Amadori A, Zamarchi R (2010) M30 neoepitope expression in epithelial cancer: quantification of apoptosis in circulating tumor cells by CellSearch analysis. Clin Cancer Res 16: 5233-5243

Sieuwerts AM, Kraan J, Bolt J, van der Spoel P, Elstrodt F, Schutte M, Martens JW, Gratama JW, Sleijfer S, Foekens JA (2009) Anti-epithelial cell adhesion molecule antibodies and the detection of circulating normal-like breast tumor cells. J Natl Cancer Inst 101: 61-66

Smith BM, Slade MJ, English J, Graham H, Luchtenborg M, Sinnett HD, Cross NC, Coombes RC (2000) Response of circulating tumor cells to systemic therapy in patients with metastatic breast cancer: comparison of quantitative polymerase chain reaction and immunocytochemical techniques. J Clin Oncol 18: 1432-1439

Stott SL, Lee RJ, Nagrath S, Yu M, Miyamoto DT, Ulkus L, Inserra EJ, Ulman M, Springer S, Nakamura Z, Moore AL, Tsukrov DI, Kempner ME, Dahl DM, Wu CL, Iafrate AJ, Smith MR, Tompkins RG, Sequist LV, Toner M, Haber DA, Maheswaran S (2010) Isolation and characterization of circulating tumor cells from patients with localized and metastatic prostate cancer. Sci Transl Med 2: 25ra23

Strati A, Markou A, Parisi C, Politaki E, Mavroudis D, Georgoulias V, Lianidou E (2011) Gene expression profile of circulating tumor cells in breast cancer by RT-qPCR. BMC Cancer 11: 422

Therasse P, Arbuck SG, Eisenhauer EA, Wanders J, Kaplan RS, Rubinstein L, Verweij J, Van Glabbeke M, van Oosterom AT, Christian MC, Gwyther SG (2000) New guidelines to evaluate the response to treatment in solid tumors. European Organization for Research and Treatment of Cancer, National Cancer Institute of the United States, National Cancer Institute of Canada. J Natl Cancer Inst 92: 205-216

Ukpo OC, Flanagan JJ, Ma XJ, Luo Y, Thorstad WL, Lewis Jr JS (2011) High-risk human papillomavirus E6/E7 mRNA detection by a novel 
in situ hybridization assay strongly correlates with p16 expression and patient outcomes in oropharyngeal squamous cell carcinoma. Am J Surg Pathol 35: 1343-1350

Van der Auwera I, Peeters D, Benoy IH, Elst HJ, Van Laere SJ, Prove A, Maes H, Huget P, van Dam P, Vermeulen PB, Dirix LY (2010) Circulating tumour cell detection: a direct comparison between the CellSearch System, the AdnaTest and CK-19/mammaglobin RT-PCR in patients with metastatic breast cancer. $\mathrm{Br}$ J Cancer 102: 276-284

Wang F, Flanagan J, Su N, Wang LC, Bui S, Nielson A, Wu X, Vo HT, Ma XJ, Luo Y (2012) RNAscope: a novel in situ RNA analysis platform for formalin-fixed, paraffin-embedded tissues. J Mol Diagn 14: 22-29
Wang Z, Zhang L, Yeung TK, Chen X (1999) Endocytosis deficiency of epidermal growth factor (EGF) receptor-ErbB2 heterodimers in response to EGF stimulation. Mol Biol Cell 10: 1621-1636

Yang J, Mani SA, Donaher JL, Ramaswamy S, Itzykson RA, Come C, Savagner P, Gitelman I, Richardson A, Weinberg RA (2004) Twist, a master regulator of morphogenesis, plays an essential role in tumor metastasis. Cell 117: 927-939

Yang Z, Zhang X, Gang H, Li X, Li Z, Wang T, Han J, Luo T, Wen F, Wu X (2007) Up-regulation of gastric cancer cell invasion by Twist is accompanied by $\mathrm{N}$-cadherin and fibronectin expression. Biochem Biophys Res Commun 358: 925-930

This work is published under the standard license to publish agreement. After 12 months the work will become freely available and the license terms will switch to a Creative Commons Attribution-NonCommercial-Share Alike 3.0 Unported License. 